\title{
Simple Models of Diabatically Forced Mesoscale Circulations and a Mechanism for Amplification
}

\author{
Alain Robichaud and Charles A. Lin
}

Department of Meteorology, McGill University, Montreal, Quebec, Canada

\begin{abstract}
A two-dimensional, linear, Boussinesq, inviscid, hydrostatic model is used to simulate the flow over an elevated diabatic source and a surface diabatic source. The elevated source is identified as being due to cooling by melting, while the surface source is associated with land/sea temperature differences. Melting frequently produces near- $0^{\circ} \mathrm{C}$ isothermal layers. The atmosphere in this case tends to have strong static stability in the melting layer, capped by a much less stable layer aloft. This creates a strong change in the vertical wave number, which might lead to resonant amplification of mesoscale perturbations, just as in mountain wave theory. Comparison with sounding data suggests that this amplification mechanism is potentially important for precipitation enhancement due to melting.
\end{abstract}

\section{INTRODUCTION}

There are many examples of mesoscale circulations in the atmosphere where diabatic forcing plays an important role. In this study we focus on simple models of melting-induced mesoscale circulations. Observational studies such as those of Atlas et al. [1969], Carbone [1982], Stewart [1984], and Stewart and King [1986] clearly demonstrate that cooling due to melting may affect the mesoscale environment significantly. Although the latent heat of fusion is about 8 times smaller than the latent heat of vaporization, melting is concentrated in a relatively shallow layer of near $-0^{\circ} \mathrm{C}$ temperature. This means that the cooling induced by melting can be comparable to that induced by evaporation. This point will be discussed in more detail later in this study.

Atlas et al. [1969] presented a detailed Doppler-radar study of the mesoscale wind perturbations induced by melting snow. They found that the spatial structure of these wind perturbations was the same as the variation in the precipitation pattern. They tried to explain the observations by modeling a hydrostatic, nonrotating inviscid atmosphere with no vertical motion. They showed that $1 \mathrm{~mm}$ of melted snow would produce a $350-\mathrm{m}$-deep $0^{\circ} \mathrm{C}$ isothermal layer and a pressure increase of $0.12 \mathrm{mbar}$ at the base of the layer, and then inferred that a "meso-high" produced the observed wind perturbations. By assuming steady state conditions, they were able to obtain horizontal wind perturbations in rough agreement with observations.

Lin and Stewart [1986] studied the steady state response of the atmosphere to prescribed temperature perturbations corresponding to typical melting environments. They used a linear, two-dimensional model, with the effects of friction and rotation included. Thermally driven circulations having a length scale similar to that of the temperature perturbations were produced. Lin and Stewart suggested that the updraft branch of this type of circulation might cause enhanced precipitation in a saturated environment. Szeto et al. $[1988 a, b]$ investigated this cooling-by-melting mechanism by using a nonlinear twodimensional numerical model. It is found that the resultant melting-induced circulations consist of a forced response resembling a gravity current and transients which are gravity

Copyright 1989 by the American Geophysical Union.

Paper number 88JD04014.

0148-0227/89/88JD-04014\$05.00 waves. Model results show that the melting associated with realistic precipitation rate can induce horizontal wind perturbations of a few meters per second and vertical motions of tens of centimeters per second. The results are also applied to three mesoscale circulations where melting often plays a significant role: rain/snow boundaries, the production of deep $0^{\circ} \mathrm{C}$ isothermal layers, and the trailing stratiform regions associated with severe squall lines.

There have been other modeling studies of mesoscale circulations forced by elevated diabatic sources. Smith and Lin [1982] used linear hydrostatic theory to examine the response of a stratified airstream to both diabatic and orographic forcings. The magnitude of the heating aloft is computed from observed rainfall rates. The phase relationships between the heating and induced vertical displacements is found to be negative. This may explain why mountain wave amplitudes are sometimes reduced in moist atmospheres. Lin and Smith [1986] explained the negative phase relationship by solving the time-dependent problem to obtain the transient approach to steady state. The vertical displacement is negative at the heating center because of the superposition of downdrafts associated with all of the regions of heated air drifting downstream. The results are applied to three problems in mesoscale dynamics: cloud interaction, heat island/orographic rain, and squall lines.

In the models referred to earlier, the influence of the vertical structure of the basic state is not considered. The main objective of this paper is to examine, using a simple model, the effects of varying static stability and wind structure in the vertical and the possible implications for melting-induced circulations. An amplification mechanism will be identified, which might be important in the enhancement of vertical velocities in diabatically induced mesoscale circulations. This mechanism is similar to that described by Klemp and Lilly [1975], who found that the strong winds near Boulder, Colorado, can be explained in terms of superposition of upward and downward propagating buoyancy waves.

The effects of combining an elevated cooling source, such as that due to melting, and a surface diabatic source are also examined. The latter source might be due to temperature contrasts between ocean and land. This combination of elevated and surface diabatic forcings would be important for a precipitation band in which melting occurred in the vicinity of a cold land/warm ocean boundary, such as the Gulf Stream regions. 
The model used and its solution, for an elevated diabatic source, are presented in section 2 . Section 3 presents the results for constant vertical wave number of the basic state. In section 4 we derive the solution for a surface diabatic source and a constant vertical wave number. Section 5 compares the relative magnitudes of different diabatic sources/sinks on the mesoscale. The amplification mechanism and effects of varying vertical wave numbers are examined in section 6. Some Canadian Atlantic Storm Project (CASP) data are given in section 7, which shows support for the amplification mechanism. Finally, section 8 contains the conclusion.

\section{Model ANd Solution}

Consider the equations describing the inviscid twodimensional flow of a perfect gas in a nonrotating system. For simplicity an incompressible Boussinesq atmosphere will be used here, although the extension to a compressible atmosphere is straightforward. The steady state governing equations are

$$
\begin{gathered}
\bar{U} \frac{\partial u^{\prime}}{\partial x}+w^{\prime} \frac{d \bar{U}}{d z}=-\frac{1}{\rho_{0}} \frac{\partial p^{\prime}}{\partial x} \\
\bar{U} \frac{\partial w^{\prime}}{\partial x}=-\frac{1}{\rho_{0}} \frac{\partial p^{\prime}}{\partial z}-\frac{g \rho^{\prime}}{\rho_{0}} \\
\frac{\partial u^{\prime}}{\partial x}+\frac{\partial w^{\prime}}{\partial z}=0 \\
\frac{\bar{U}}{\rho_{0}} \frac{\partial \rho^{\prime}}{\partial x}=\frac{N^{2} w^{\prime}}{g}-\frac{J}{C_{p} \bar{T}}
\end{gathered}
$$

where $N^{2}$ is the Brunt-Väisälä frequency squared $(g / \theta)(d \theta / d z)$ and $J$ is the heating (or cooling) rate of the diabatic source. The notation used is standard, and a list of symbols is presented in the notation section at the end of the paper. Equations (1) and (2) represent momentum equations in $x$ and $z$, while (3) and (4) are the continuity and thermodynamic equations, respectively. This set of equations is the same as that used by Smith and Lin [1982], except that we now allow for variation of $\bar{U}$ and $N$ in the vertical.

We may combine the equations to obtain the vertical velocity equation

$$
\frac{\partial^{2} w^{\prime}}{\partial x^{2}}+\frac{\partial^{2} w^{\prime}}{\partial z^{2}}+\left(\frac{N^{2}}{\bar{U}^{2}}-\frac{1}{\bar{U}} \frac{\partial^{2} \bar{U}}{\partial z^{2}}\right) w^{\prime}=\frac{g J}{C_{p} \bar{T} \bar{U}^{2}}
$$

Equation (5) is a Boussinesq form of the forced TaylorGoldstein equation [Raymond, 1984]. The simplest solution to (5) is obtained by assuming a constant vertical wave number $m$, where

$$
m^{2}=\frac{N^{2}}{\bar{U}^{2}}-\frac{1}{\bar{U}} \frac{\partial^{2} \bar{U}}{\partial z^{2}}
$$

Since we consider horizontal scales of more than a few kilometers, the hydrostatic approximation is used; this is equivalent to assuming

$$
\frac{\partial^{2} w^{\prime}}{\partial z^{2}} \gg \frac{\partial^{2} w^{\prime}}{\partial x^{2}}
$$

Using (6) and (7), we then have from (5) (after dropping primes)

$$
\frac{\partial^{2} w}{\partial z^{2}}+m^{2} w=\frac{g J}{C_{p} \bar{T} \bar{U}^{2}}
$$

Equation (8) may be solved for a finite depth heat source by using the Green's function method, as done by Smith and Lin [1982]. However, here we will solve the problem with the Fourier transform method. This is simpler mathematically for the type of vertical heating profile that we are dealing with in this paper.

We consider a three-layer piecewise constant model for $m$ and assume that the structure of the diabatic source $J$ is separable in $z$ and $x$.

$$
J=J_{0} F(z) G(x)
$$

$$
\begin{array}{cc}
F(z)=\sin M\left(z-h_{0}\right) & h_{0}<z<H \\
F(z)=0 & \text { otherwise } \\
M=\pi /\left(H-h_{0}\right)
\end{array}
$$

The horizontal structure function $G(x)$ is given by the bellshaped function

$$
G(x)=a^{2} /\left(a^{2}+x^{2}\right)
$$

We express the Fourier transforms of the vertical velocity $w$ and $G$ as follows:

$$
\begin{aligned}
\tilde{w}(k, z) & =\frac{1}{\pi} \int_{-\infty}^{\infty} w(x, z) \exp (-i k x) d k \\
\tilde{G}(k) & =\frac{1}{\pi} \int_{-\infty}^{\infty} G(x) \exp (-i k x) d k
\end{aligned}
$$

Equation (8) then becomes

$$
\frac{d^{2} \tilde{w}}{d z^{2}}=m^{2} \tilde{w}=\frac{g J_{0} \tilde{G}(k) F(z)}{C_{p} \bar{T} \bar{U}^{2}}
$$

with $\tilde{G}(k)$ being the Fourier transform $(x \rightarrow k)$ of $(11)$; that is

$$
G(k)=a \exp (-k a)
$$

The function $G(x)$ simulates a localized diabatic source in $x$, and $F(z)$ has a sinusoidal shape. The latter is a good approximation to the cooling rate due to melting [Szeto et al., 1988a]. A schematic sketch of this vertical structure is shown in Figure 1. The diabatic source acts in the middle layer (between $h_{0}$ and $H$ ) and is zero for $z \geq H$ and $z \leq h_{0}$.

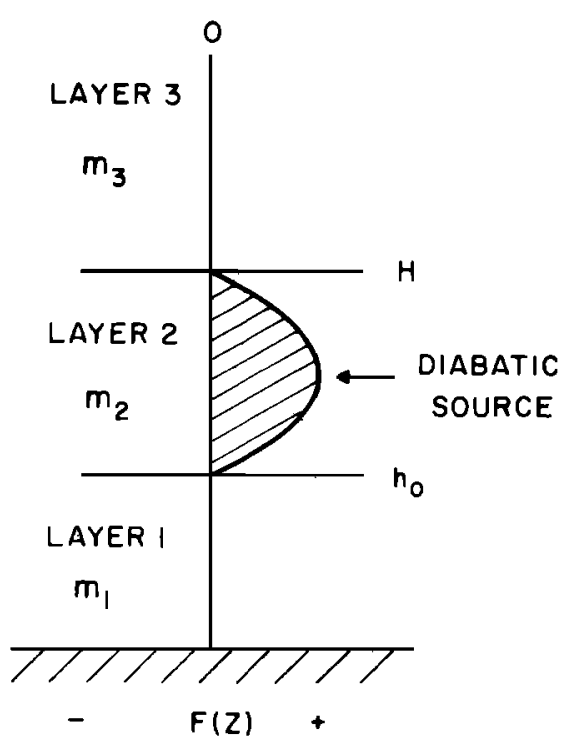

Fig. 1. Piecewise constant three-layer model for the vertical wave number $m$. The elevated heat sink is located between heights $h_{0}$ and $H$ and has a sinusoidal form with scale $M$. 
We obtain the solution of (12) for the Fourier-transformed vertical velocities in the three layers $\left(\tilde{w}_{1}, \tilde{w}_{2}, \tilde{w}_{3}\right)$ as

$$
\begin{gathered}
\tilde{w}_{1}=\tilde{A} \exp \left(i m_{1} z\right)+\tilde{B} \exp \left(-i m_{1} z\right) \quad z<h_{0} \\
\tilde{w}_{2}=\tilde{C} \exp \left(i m_{2} z\right)+\tilde{D} \exp \left(-i m_{2} z\right) \\
+\frac{g J_{0} \tilde{G}(k) \sin M\left(z-h_{0}\right)}{C_{p} \bar{T} \bar{U}^{2}\left(m_{2}{ }^{2}-M^{2}\right)} \quad h_{0} \leq z \leq H \\
\tilde{w}_{3}=\tilde{E} \exp \left(i m_{3} z\right)+\tilde{F} \exp \left(-i m_{3} z\right) \quad z>H
\end{gathered}
$$

Notice that coefficients $\tilde{A}, \tilde{B}, \tilde{C}, \tilde{D}, \tilde{E}, \tilde{F}$ are dependent on the Fourier wave number $k$ and are complex quantities. The solution for layers 1 and 3 is simply the homogeneous solution of (12), whereas in layer $2\left(h_{0} \leq z \leq H\right)$ the complete solution is the sum of the homogeneous solution plus a particular solution which depends on the diabatic forcing profile.

Since the set of equations (14) contains six unknown coefficients, we need six independent boundary conditions. These are

Condition 1

$$
\tilde{w}_{1}=0 \quad z=0
$$

Condition 2

$$
\tilde{w}_{3} \sim \exp \left(i m_{3} z\right)
$$

Condition 3

$$
\tilde{w}_{1}=\tilde{w}_{2} \quad z=h_{0}
$$

Condition 4

$$
\tilde{w}_{2}=\tilde{w}_{3} \quad z=H
$$

Condition 5

$$
\frac{\partial \tilde{w}_{1}}{\partial z}=\frac{\partial \tilde{w}_{2}}{\partial z} \quad z=h_{0}
$$

Condition 6

$$
\frac{\partial \tilde{w}_{2}}{\partial z}=\frac{\partial \tilde{w}_{3}}{\partial z} \quad z=H
$$

Conditions 1 and 2 represent free slip boundary conditions at the ground and upper radiation condition, respectively, Conditions 3 and 4 express continuity of vertical velocities at both interface $h_{0}$ and $H$, while 5 and 6 represent the continuity of pressure at both $h_{0}$ and $H$. (See Appendix A for more details.) This method for solving (12) is similar to that used by Hayashi [1976], although the physical problem examined there is somewhat different. The final solutions, after using the six boundary conditions and taking the inverse Fourier transform, are

$$
\begin{gathered}
w_{1}(x, z)=\operatorname{Real}\left\{\frac{\sin m_{1} z}{\sin m_{1} h_{0}}\left[C \exp \left(i m_{2} h_{0}\right)+D \exp \left(-i m_{2} h_{0}\right)\right]\right\} \\
w_{2}(x, z)=\operatorname{Real}\left\{C \exp \left(i m_{2} z\right)+D \exp \left(-i m_{2} z\right)\right. \\
\left.+\frac{J_{0} G(x) \sin M\left(z-h_{0}\right)}{m_{2}^{2}-M^{2}}\right\}
\end{gathered}
$$

$w_{3}(x, z)=R \operatorname{eal}\left\{\exp \left[i m_{3}(z-H)\right]\left[C \exp \left(i m_{2} H\right)+D \exp \left(-i m_{2} H\right)\right]\right\}$ with

$$
\begin{gathered}
C=\beta / \Delta \\
\beta=M Q\left\{\sin m_{1} h_{0}-i\left[\frac{m_{1} \cos m_{1} h_{0}+i m_{2} \sin m_{1} h_{0}}{m_{2}+m_{3}}\right]\right. \\
\left.\cdot \exp \left[i m_{2}\left(H-h_{0}\right)\right]\right\} \\
\Delta=\left(m_{2}{ }^{2}-M^{2}\right)\left\{\left(m_{1} \cos m_{1} h_{0}-i m_{2} \sin m_{1} h_{0}\right) \exp \left(i m_{2} h_{0}\right)\right. \\
\left.+\alpha\left(m_{1} \cos m_{1} h_{0}+i m_{2} \sin m_{1} h_{0}\right) \exp \left[i m_{2}\left(2 H-h_{0}\right)\right]\right\} \\
D=\alpha C \exp \left(2 i m_{2} H\right)+\frac{i M Q \exp \left(i m_{2} H\right)}{\left(m_{2}+m_{3}\right)\left(m_{2}{ }^{2}-M^{2}\right)} \\
Q=\frac{J_{0} a g(a+i x)}{\left(a^{2}+x^{2}\right) C_{p} \bar{T} \bar{U}^{2}} \\
\alpha=\frac{m_{2}-m_{3}}{m_{2}+m_{3}}
\end{gathered}
$$

Note that the quantity $\alpha$ represents the coefficient of reflection of vertically propagating gravity waves at the interface $H$ between a top layer having a vertical wave number $m_{3}$ and a middle layer having a vertical wave number $m_{2}$. If $\alpha$ is greater than zero (but less than 1), there is partial reflection at the interface $z=H$, which may lead to significant amplification (resonance). This is described in a later section. A major difference between our model for diabatically forced circulations and some of the studies in the literature [e.g., Hayashi, 1976; Smith and Lin, 1982] is that the latter assume no vertical variation of the wave number $m$. A reflective level is thus absent in these studies.

\section{Results for Constant Vertical Wave Number}

The airflow described by solution (16) is presented below for constant vertical wave number $\left(m_{1}=m_{2}=m_{3}\right)$. The effects of vertically varying $m$ are considered in section 6 .

To obtain physically acceptable solutions, we will include in all computations a compensative diabatic source to avoid the problem of net heating [Smith and Lin, 1982]. That is, instead of (11), we write

$$
G(x)=a\left\{\frac{a}{a^{2}+x^{2}}-\frac{a_{0}}{a_{0}{ }^{2}+x^{2}}\right\} \quad a_{0} \gg a
$$

This ensures that the net heating at any level $z$ is zero:

$$
\int_{-\infty}^{\infty} G(x) d x=0
$$

Including the second term in (21) results in very little change in the circulation near the region of interest. It is an alternative to including the effects due to radiative processes or friction that actually occur downstream of the diabatic source. Failure to include such a term in (21) leads to an unbounded solution for the stream function at $x=\infty$, that is, in the direction of the mean wind. A generalized discussion of this problem, using a group velocity argument, is given by Bretherton [1988].

Figure 2 depicts the vertical motion over a strong cooling source (e.g., melting, evaporation) located between the ground and $2 \mathrm{~km}$ (i.e., $h_{0}=0$ and $H=2 \mathrm{~km}$ ). The maximum cooling occurs at $z=1 \mathrm{~km}$ and $x=0$, with a horizontal half width $a=10 \mathrm{~km}$, and $a_{0}=100 \mathrm{~km}$.

We see maximum positive vertical velocity just upstream and above the point where the maximum cooling occurs. The maximum downdraft occurs $4 \mathrm{~km}$ right above the location of 


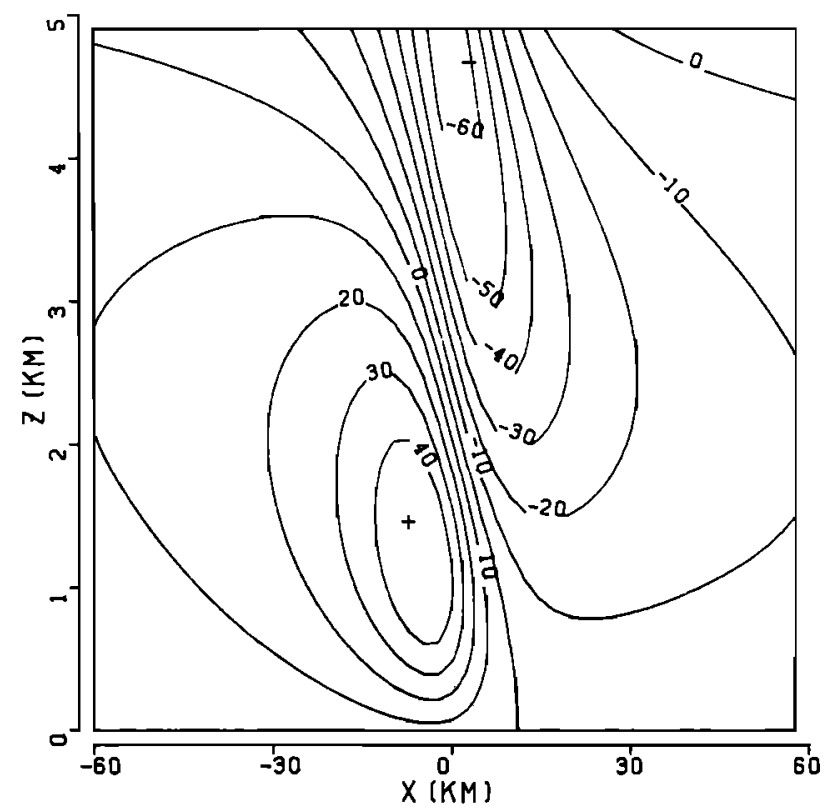

Fig. 2. Vertical motion (in centimeters per second) over an elevated heat sink centered at $x=0, z=1 \mathrm{~km}$, with depth $2 \mathrm{~km}\left(h_{0}=0\right.$, $H=2 \mathrm{~km}$ ). A sinusoidal structure is assumed in the vertical, and a bell-shaped structure with scale $a=10 \mathrm{~km}$ is assumed in the horizontal. The maximum amplitude of the cooling source is $-4 \mathrm{~J} \mathrm{~kg}^{-1}$; other parameters are $U=15 \mathrm{~m} \mathrm{~s}^{-1}, m=0.67 \mathrm{~km}^{-1}$, and $T=273^{\circ} \mathrm{K}$.

the maximum cooling. Note that the extrema of the upward displacement, $\zeta_{m}$, occurs where $w=0$, as $w=U \partial \zeta / \partial x$. There is thus a region of maximum upward displacement located just downstream of the point of maximum cooling. This occurs because, in the steady state, the flow adjusts itself in the presence of mean horizontal advection to produce a negativephase relationship between the diabatic forcing and the upward displacement field [Raymond, 1972; Smith and Lin, 1982]. It is worth noting that our results are in qualitative agreement with those of Lin and Smith [1986], who developed a time-dependent solution of the same problem with a slightly

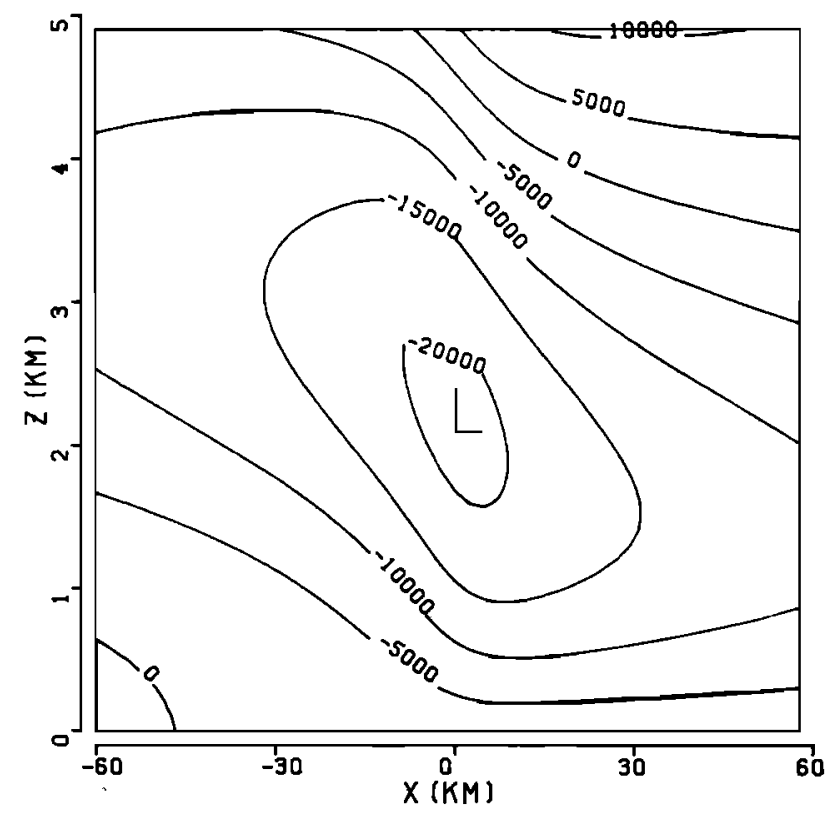

Fig. 3. Perturbation stream function (in square meters per second) over the elevated heat sink of Figure 2.

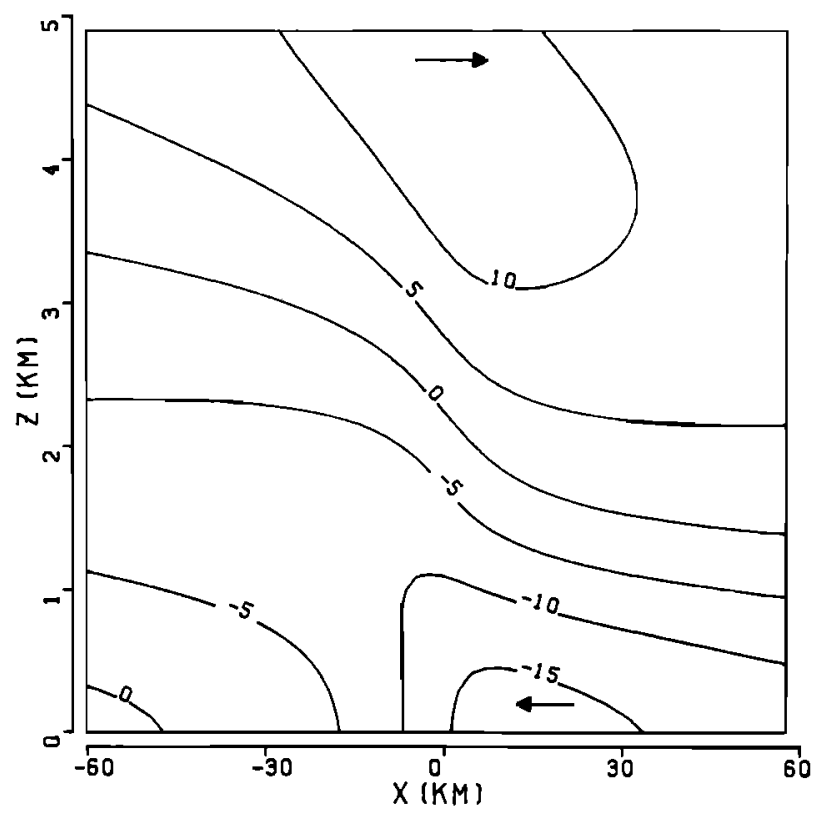

Fig. 4. Perturbation wind speed $u^{\prime}$ (in centimeters per second) over the elevated heat sink of Figure 2.

different vertical structure of the diabatic forcing. Our steady state solution (Figure 2) gives good qualitative agreement when compared to their solution for large times (see Figure $9 d$ of their paper). Note in Figure 2 that the phase relation between $w$ and $J$ is also negative. We show in Appendix B that as the thermally induced Froude number tends to zero, the vertical motion and the heating become in phase.

Figure 3 shows the stream function, obtained from the vertical velocity by integration

$$
\Psi(x, z)=-\int_{-\infty}^{x} w d x
$$

The maximum stream function value is located at $x=0$ and just above the cooling layer, which extends from 0 to $2 \mathrm{~km}$. Figure 4 shows the solution for $u$, the wind perturbation obtained from $\Psi$ by differentiation with respect to $z$.

$$
u=\frac{\partial \Psi}{\partial z}
$$

We see that the wind shear is maximum for slightly positive $x$ values in the diabatic layer $(z<H)$. A possible qualitative comparison is with the results of Stewart [1984], who noticed that strong wind shears exist across a melting layer. These wind shears may in fact be partly the result of the diabatic forcing. Expressions for the density perturbation are easily derived from the thermodynamic equation (4):

$$
\begin{gathered}
\rho(x, z)=-\frac{N^{2} \Psi \rho_{0}}{\bar{U} g} \quad z \geq H \\
\rho(x, z)=-\frac{N^{2} \Psi \rho_{0}}{\bar{U} g}-\left\{\frac{\rho_{0} J_{0} F(z)}{\bar{U} C_{p} \bar{T}} \int G(x) d x\right\} \quad z<H
\end{gathered}
$$

Figure 5 shows the solution; it consists of a mesohigh in the downdraft region within the cooling layer (at $x=25 \mathrm{~km}$, $z=1.2 \mathrm{~km}$ ). Note that we may regard the solution obtained here as due to a steady cooling source located at $x=0$, in the frame of reference moving at speed $-\vec{U}$. That is, Figure 5 might represent a leftward moving squall line with the meso- 


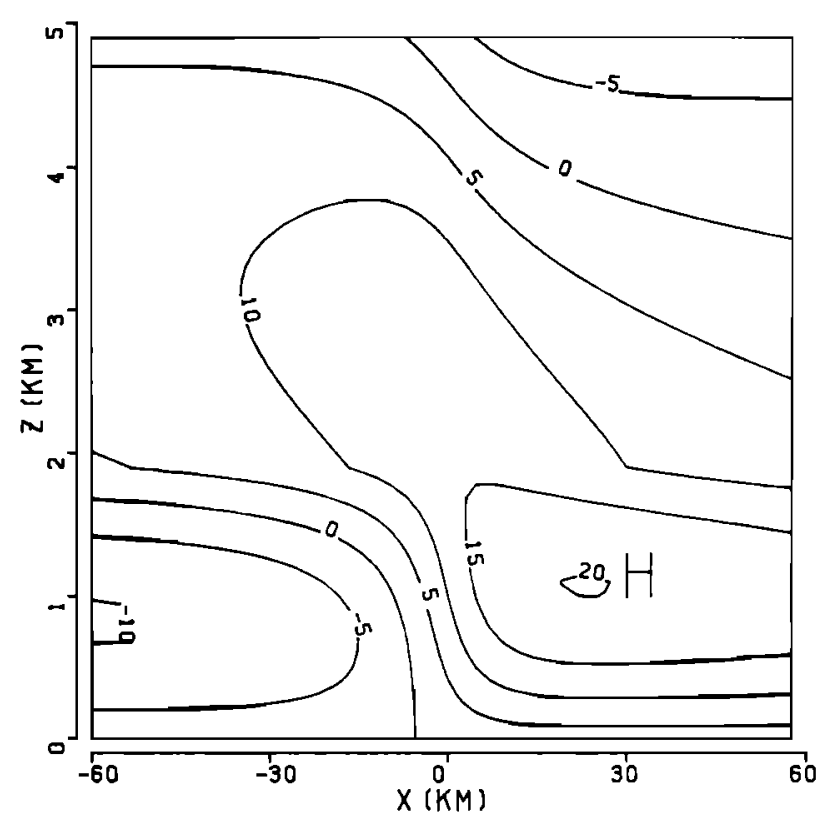

Fig. 5. Perturbation density $\left(10^{-3} \mathrm{~kg} \mathrm{~m}^{-3}\right)$ over the elevated heat sink of Figure 2.

high behind, at $x>0$. Pressure and density perturbations are related through the hydrostatic equation; the latter then implies that a mesohigh pressure area is located below the region of maximum density perturbation in Figure 5, near $x=30 \mathrm{~km}$. Studies such as those of Fujita [1959], Fernandez [1982], Lin and Smith [1986], and others noted such mesohighs behind squall lines, which might be caused by melting and evaporation. The strong horizontal density gradient near $x=0$ below $2 \mathrm{~km}$ could be associated with the gust front observed immediately in front of moving squall lines.

\section{Solution for a Surface Diabatic Forcing}

We now examine the effects of a stationary surface diabatic source such as that due to land/sea temperature contrast. We assume a diabatic source whose maximum strength is located at the surface and which decreases linearly with height to zero at $z=h_{0}$ (Figure 6).

$$
\begin{array}{ll}
J=J_{0} G_{s}\left(1-z / h_{0}\right) & z \leq h_{0} \\
J=0 & z>h_{0}
\end{array}
$$

Other functions could be used to simulate the variation of heating in the vertical [e.g., Rotunno, 1983], but we choose (26) for mathematical simplicity. This simulates the vertical transport of heat and permits a straightforward solution of (12). We take for the horizontal structure of the diabatic forcing

$$
G_{s}(x)=2 c x\left\{\frac{1}{x^{2}+c^{2}}-\frac{1}{x^{2}+c_{0}^{2}}\right\}
$$

where $c$ and $c_{0}$ are the scales of the heating lunction and compensative source, respectively. Notice that (27) gives a bounded stream function, whereas the first term of (27) alone does not. The boundary conditions remain as before.

The solution of (12) for a three-layer piecewise constant model for the vertical wave number $m$, for a surface diabatic source with this spatial structure, is

$$
\begin{gathered}
\tilde{w}_{1}(k, z)=\tilde{A}_{s} \exp \left(i m_{1} z\right)+\widetilde{B}_{s} \exp \left(-i m_{1} z\right) \\
+\frac{g J_{0} \tilde{G}_{s}(k)\left(1-z / h_{0}\right)}{C_{p} \bar{T} \bar{U}^{2} m_{1}{ }^{2}} \quad z \leq h_{0} \\
\tilde{w}_{2}(k, z)=\tilde{C}_{s} \exp \left(i m_{2} z\right)+\tilde{D}_{s} \exp \left(-i m_{2} z\right) \quad h_{0}<z<H \\
\tilde{w}_{3}(k, z)=\tilde{E}_{s} \exp \left(i m_{3} z\right) \quad z \geq H
\end{gathered}
$$

with

$$
\begin{gathered}
\tilde{A}_{s}=-\frac{1}{2}\left[\frac{i \tilde{Q}(0) \exp \left(-i m_{1} h_{0}\right)\left(m_{1}+m_{2} \gamma\right)+(\partial \tilde{Q} / \partial z)}{m_{2} \gamma \sin m_{1} h_{0}+i m_{1} \cos m_{1} h_{0}}\right] \\
\tilde{B}_{s}=-\left(\tilde{A}_{s}+\tilde{Q}(0)\right) \\
\tilde{C}_{s}=\left\{\frac{\tilde{A}_{s} \exp \left(i m_{1} h_{0}\right)+\tilde{B}_{s} \exp \left(-i m_{1} h_{0}\right)}{1+\alpha \exp \left(i 2 m_{2}\right)\left(H-h_{0}\right)}\right\} \exp \left(-i m_{2} h_{0}\right) \\
\tilde{D}_{s}=\tilde{C}_{s} \alpha \exp \left(2 i m_{2} H\right) \\
\tilde{E}_{s}=\tilde{C}_{s}(1+\alpha) \exp \left[i\left(m_{2}-m_{3}\right) H\right]
\end{gathered}
$$

and

$$
\begin{gathered}
\gamma=\left\{1-\alpha \exp \left[2 i m_{2}\left(H-h_{0}\right)\right]\right\} /\left\{1+\alpha \exp \left[2 i m_{2}\left(H-h_{0}\right)\right]\right\} \\
\widetilde{Q}(0)=\frac{g J_{0} \tilde{G}_{s}(k)}{C_{p} \bar{T} \bar{U}^{2} m_{1}^{2}} \\
\frac{\partial \tilde{Q}}{\partial Z}=-\frac{g J_{0} \tilde{G}_{s}(k)}{h_{0} C_{p} \bar{T} \bar{U}^{2} m_{1}{ }^{2}} \\
\widetilde{G}_{s}(k)=2 i \pi c\left\{\exp (-c k)-\exp \left(-c_{0} k\right)\right\}
\end{gathered}
$$

$\tilde{G}_{s}(k)$ is the Fourier transform $(x \rightarrow k)$ of (27). Analytical solutions in real space are easily obtained by taking the inverse Fourier transform of (28). The horizontal variation of the heating is illustrated in Figure 7. There is cooling for $x<0$ and warming for $x>0$, which could simulate an idealized land/sea temperature contrast, with the coast located at $x=0$.

The result for the case where $m_{1}=m_{2}=m_{3}$ is shown in Figure 8; a negative phase relation is again obtained between heating and vertical motion. This is in agreement with Smith and $\mathrm{Lin}$ [1982], and more recently with $\mathrm{Hsu}$ [1987]. The latter also shows that as the scale of the heating increases, this nega-

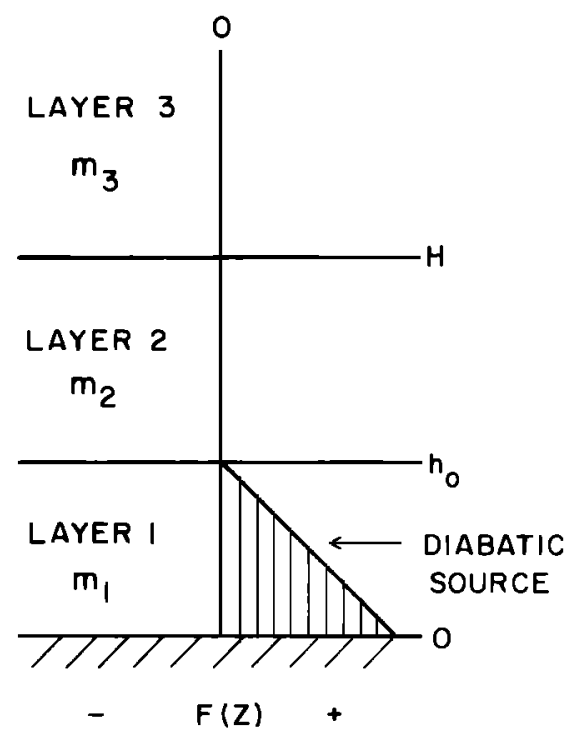

Fig. 6. Vertical structure function $F(z)$ for the surface diabatic forcing. 
HEATING RATE (W/KG) VS DISTANCE (KM)

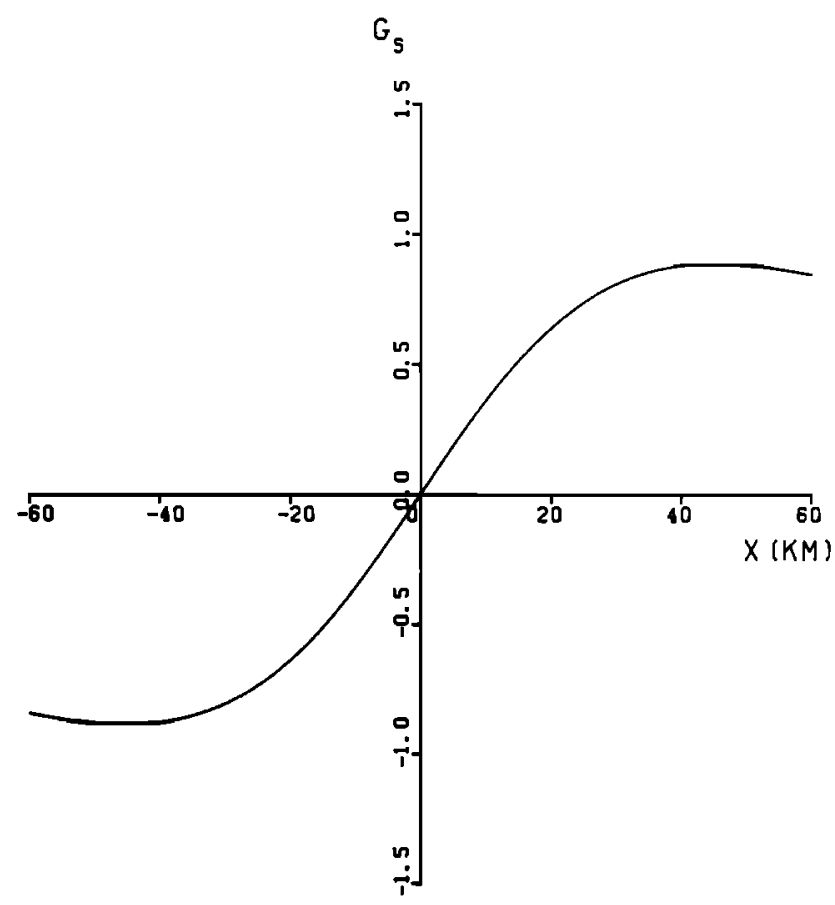

Fig. 7. Horizontal structure function of an idealized surface heating distribution simulating land/sea contrast, with cooling over land $(x<0)$ and warming over ocean $(x>0)$. The coastline is located at $x=0$.

tive phase relation no longer holds and heating becomes positively correlated with vertical velocity.

Figures 9 and 10 show the associated stream function and the perturbation density, respectively. The maximum density perturbation is located where the heating changes sign. This is consistent with $(25 b)$ as the density perturbation is related to the horizontal integral of the heating profile. The second term

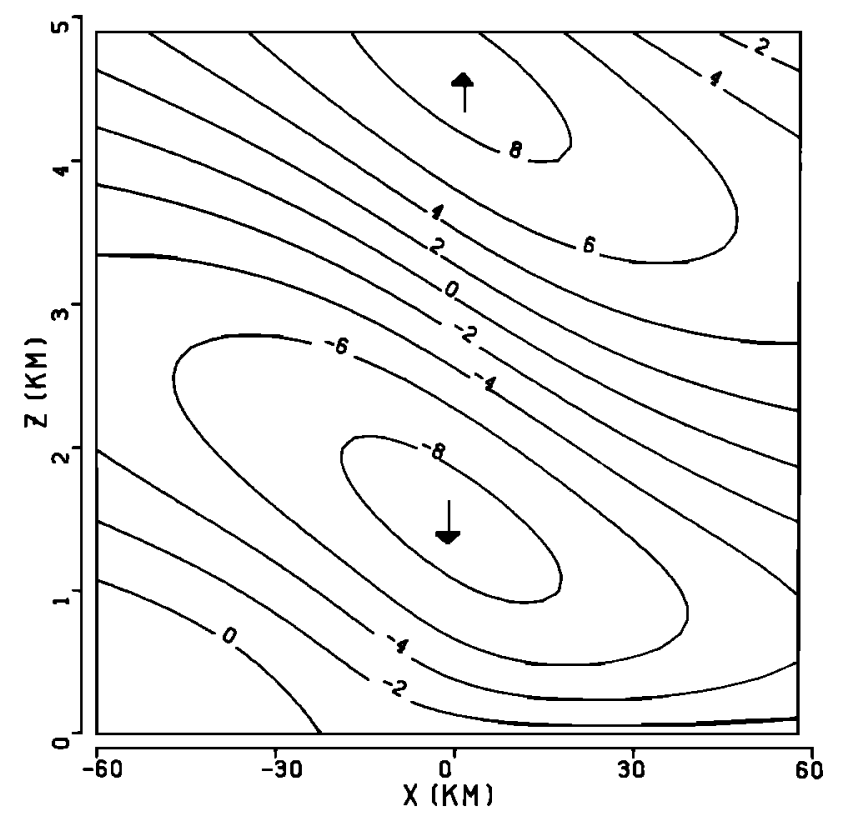

Fig. 8. Perturbation vertical motion (in centimeters per second) over surface diabatic forcing of Figure 6. The parameter values are $m=1.0 \mathrm{~km}^{-1}, c=50 \mathrm{~km}, c_{0}=200 \mathrm{~km}, \bar{U}=15 \mathrm{~m} \mathrm{~s}^{-1}$, and $\bar{T}=$ $273^{\circ} \mathrm{K}$.

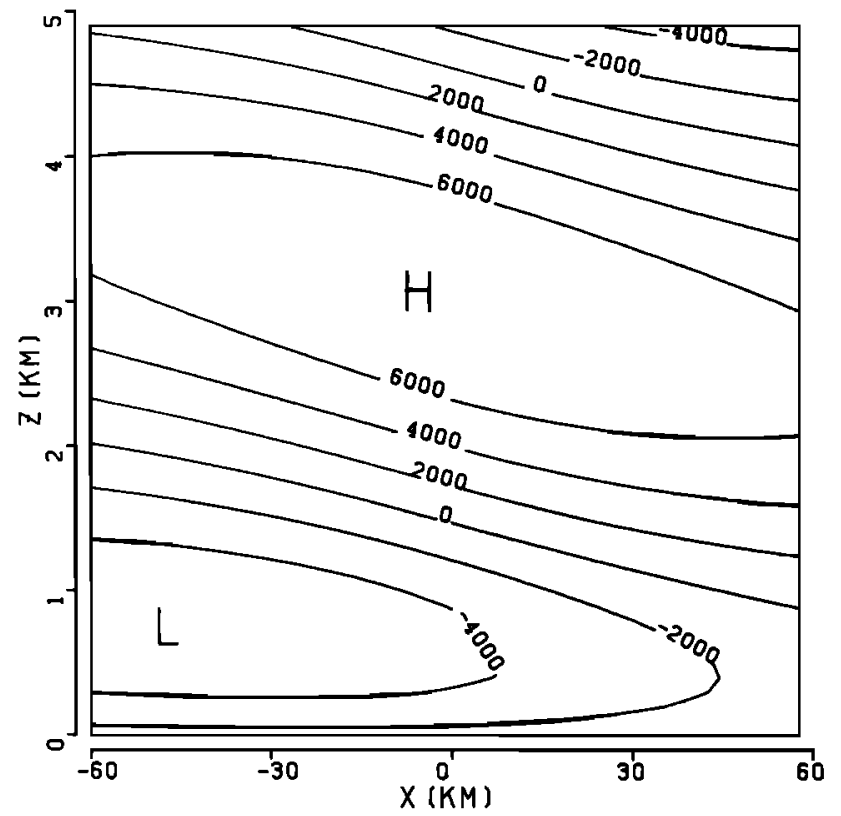

Fig. 9. As in Figure 8, but for the stream function (in square meters per second).

thus has its maximum value where $G(x)$ changes from cooling to warming. Note also that the perturbation stream function is exactly out of phase with the perturbation density over the heating layer, in agreement with (25a).

The effects of varying the vertical structure of $m$ will be discussed in section 6 .

\section{A Comparison of Surface and Elevated Diabatic Forcings}

Precipitation bands frequently occur in the presence of near-isothermal $0^{\circ} \mathrm{C}$ layers [Stewart, 1984], which suggests that these circulations might be associated with elevated melting-induced diabatic forcings. Szeto et al. [1988a], using a nonlinear two-dimensional numerical model, showed that or-

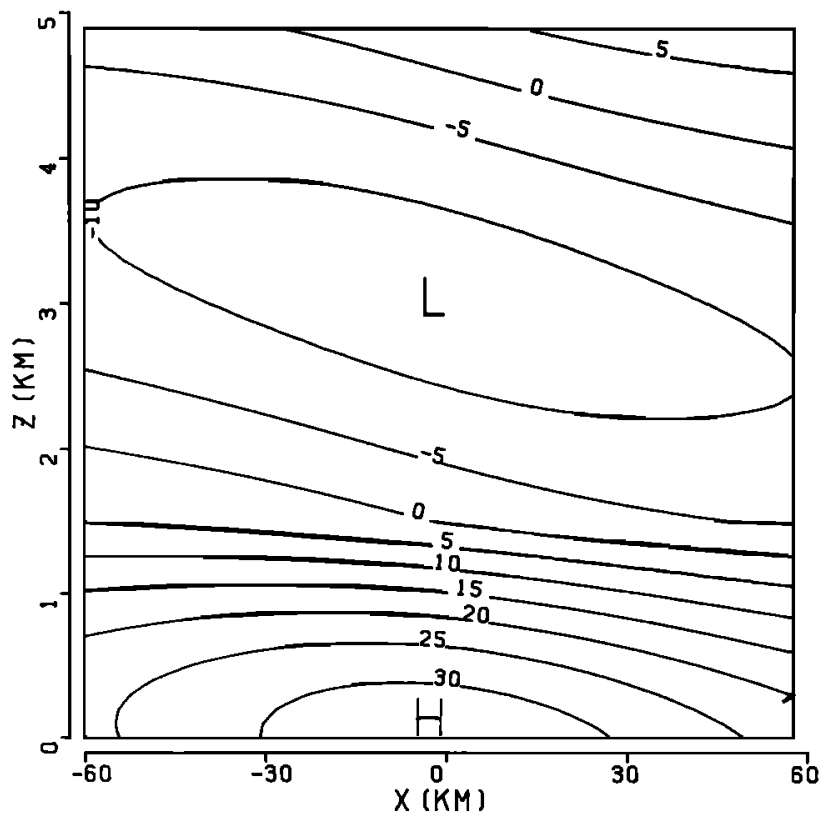

Fig. 10. As in Figure 8, but for the perturbation density $\left(10^{-3} \mathrm{~kg}\right.$ $\mathrm{m}^{-3}$ ). 
ganized mesoscale circulations can indeed result from this elevated diabatic forcing. A surface heat source would also be present, when a melting-induced circulation develops across a land/sea boundary, such as that associated with the Gulf Stream. In our linear model we may simply add the solutions obtained from elevated melting-induced $\left(w_{m}\right)$ and surface $\left(w_{s}\right)$ diabatic forcings to yield the total vertical motion field

$$
w=w_{m}+w_{s}
$$

with $w_{m}$ and $w_{s}$ given by (16) and (28), respectively. Although this linear superposition yields no additional physical insight, it is interesting to compare the relative magnitudes of the responses due to both types of diabatic forcings. The amplitude of the cooling due to melting $\left(J_{m}\right)$ may be expressed as

$$
J_{m}=\frac{L_{f} P_{0}}{d_{m}}
$$

where $\boldsymbol{P}_{0}$ is the rate of the background precipitation, which is assumed to melt over the distance $d_{m} ; L_{f}$ is the latent heat of fusion. The amplitude of the surface melting $\left(J_{s}\right)$ may be represented as

$$
J_{s}=\frac{H_{0}}{\rho_{0} h_{0}}
$$

where $H_{0}$ is the surface heat flux, assumed to be distributed over a vertical scale $h_{0} ; \rho_{0}$ is the air density. A typical value for the heat flux might be $H_{0}=250 \mathrm{~W} \mathrm{~m}^{-2}$ over the Gulf Stream region, distributed over a distance $h_{0}=500 \mathrm{~m}$. Typical melting layer depths are $d_{\mathrm{m}}=500 \mathrm{~m}$ from both observations [Stewart, 1984] and modeling results [Szeto et al., 1988a]. The ratio $J_{m} / J_{s}$ is then unity for a precipitation rate of about $5 \mathrm{~mm}$ $\mathrm{h}^{-1}$. Thus on the mesoscale under typical conditions, melting may induce circulations of comparable amplitudes to those generated by surface diabatic sources associated with land/sea differences.

The amplitude of melting-induced cooling can also be comparable to that due to evaporation $\left(J_{e}\right)$. The latter may be expressed as

$$
J_{e}=\frac{L_{v} f P_{0}}{d_{e}}
$$

where $P_{0}$ is the precipitation rate and $f$ is the fraction undergoing evaporation over the distance $d_{e} ; L_{v}$ is the latent heat of fusion. A measure of the relative cooling magnitudes of melting to evaporation is thus the ratio

$$
\frac{J_{m}}{J_{e}}=\frac{L_{f}}{L_{v}} \frac{d_{e}}{d_{m}} \frac{1}{f}
$$

Typical values are $L_{f} / L_{v}=1 / 8, d_{m}=500 \mathrm{~m} ;$ Atlas et al. [1969], in their study of melting effects in precipitation band formation, give $f=0.2$ for $d_{e}=1.5 \mathrm{~km}$. This ratio is then of order unity, suggesting that melting effects are comparable to evaporative cooling.

Our simple estimates of this section thus suggest that melting as a diabatic forcing in mesoscale systems should not be neglected. Both observation [Stewart, 1984] and modeling studies [Szeto et al., 1988a, b] suggest that melting effects could give rise to organized mesoscale circulations of significant amplitudes. In section 6 we examine the effects of varying vertical wave number in our linear model. In particular, we investigate an amplification mechanism through which the response to melting-induced cooling may be enhanced.
TABLE 1. Amplitude of the Maximum Vertical Velocity Below the Interface $z=H$, as a Function of the Reflection Coefficient $\alpha$

\begin{tabular}{lccc}
\hline$\alpha$ & $w_{\text {max }}, \mathrm{cm} \mathrm{s}^{-1}$ & $A$ & $m_{3}, \mathrm{~km}^{-1}$ \\
\hline 0 & 2.8 & 1.0 & 1.57 \\
0.1 & 3.1 & 1.1 & 1.28 \\
0.2 & 3.5 & 1.25 & 1.05 \\
0.3 & 4.2 & 1.5 & 0.85 \\
0.4 & 5.2 & 1.9 & 0.67 \\
0.5 & 6.5 & 2.3 & 0.50 \\
0.6 & 8.6 & 3.1 & 0.39 \\
0.7 & 12.1 & 4.3 & 0.28 \\
0.8 & 19.1 & 6.8 & 0.17 \\
\hline
\end{tabular}

$A$ is the amplification factor relative to the case $\alpha=0 ; m_{3}$ is the vertical wave number in the top layer; maximum vertical velocity is $w_{\max }$.

\section{Results for Varying Vertical Wave Number} AND AN AMPLIFICATION MeChaNISM

We now examine the effects of varying vertical wave number. In particular, we seek the possibility of perturbation amplification due to vertical nonuniformity. Inspection of the expression for the coefficient $C$ for the vertical velocity in (17) shows that there are two possibilities for the denominator to vanish. The more obvious one has $m_{2}=M$. The other possibility will be discussed later in this section.

When the vertical scale of the wave in the second layer $\left(\pi / m_{2}\right)$ coincides with the vertical scale of the forcing $(\pi / M)$, a large perturbation amplitude results. If $m=m_{1}=m_{2}=m_{3}$ and $h_{0}=0$ in (17), we recover the solution presented by Hayashi [1976]. In that study, Hayashi shows that the solution becomes indeterminate and a bounded solution is obtained using l'Hospital's rule. The solution has maximum vertical velocity just above the level of maximum diabatic forcing;

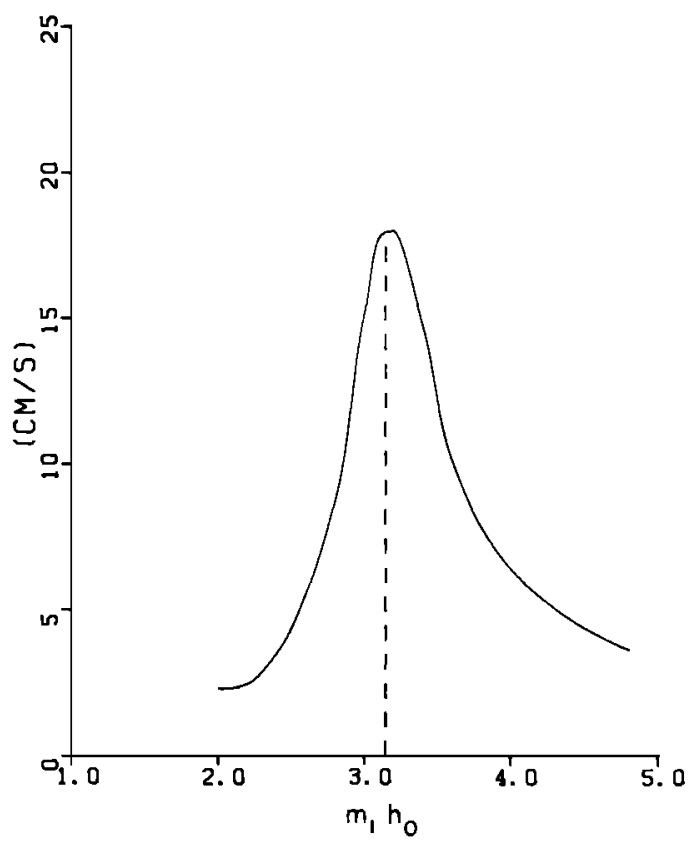

Fig. 11. Amplitude of vertical velocity (in centimeters per second) in the region below $z=H$, as a function of the nondimensional vertical wave number of the lowest layer $\left(m_{1} h_{0}\right)$, over an elevated cooling source. The parameter values are $m_{1}=2.0 \mathrm{~km}^{-1}, m_{2}=\pi / 2 \mathrm{~km}^{-1}$, $\alpha=0.75$, and $H-h_{0}=1 \mathrm{~km}$. 

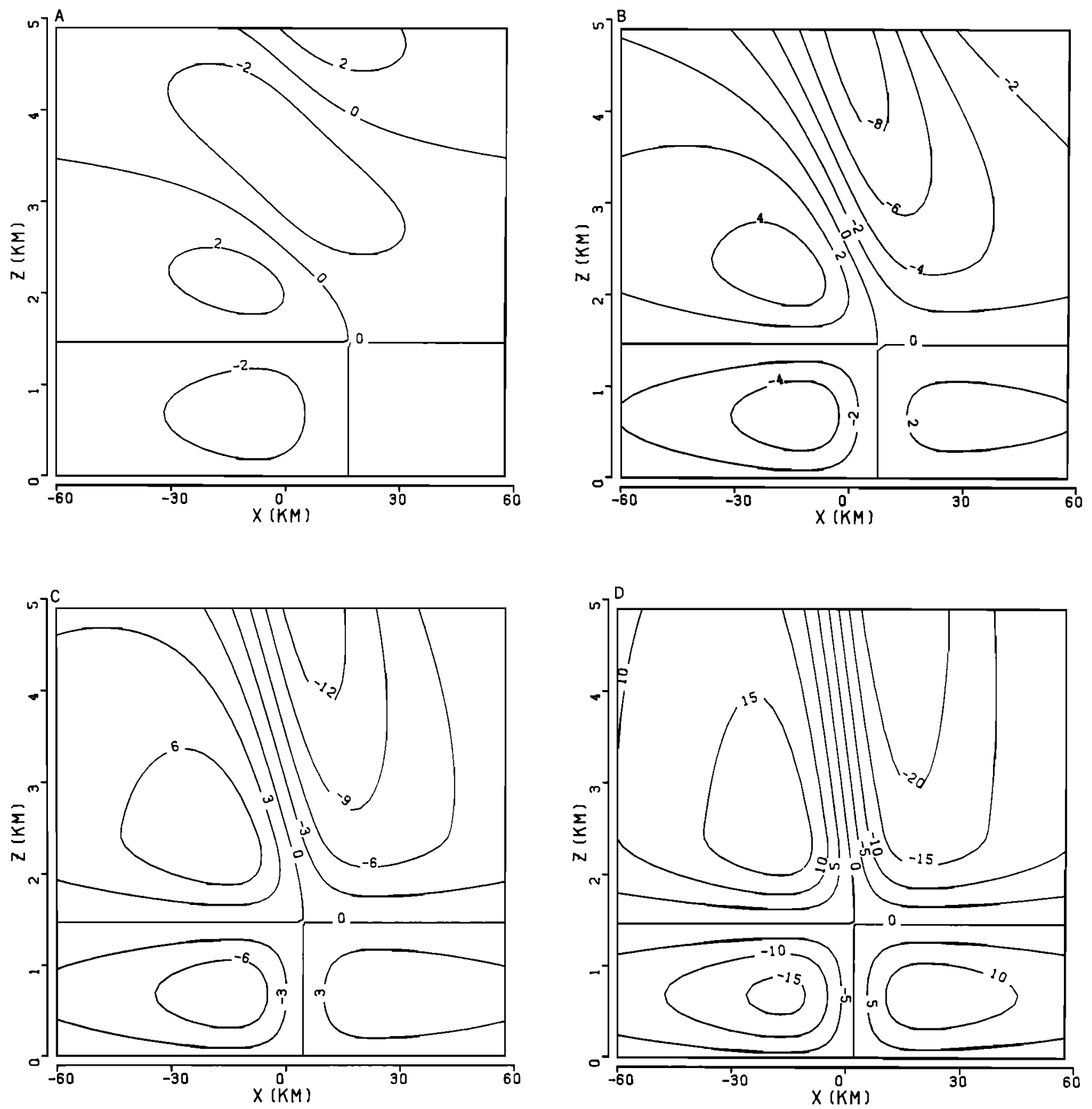

Fig. 12. Vertical velocity profiles (in centimeters per second) as a function of height for different values of the reflection coefficient : (a) $\alpha=0,(b) \alpha=0.4,(c) \alpha=0.6$, and $(d) \alpha=0.8$.

Hayashi refers to this solution as "non-singular resonance." However, this type of solution with a bounded maximum is due to the sinusoidal nature of the vertical structure function $F(z)$ of the diabatic forcing and is not associated with variation of vertical wave number with height, or with wave trapping. In Appendix $\mathrm{C}$ we show the solution for a nonsinusoidal structure function $F(z)$, where no amplification of this type occurs.

We examine instead the other possibility of "resonance," which is due to the presence of at least one reflective level or a discontinuity in the vertical wave number $m$. This resonance condition obtains when the other part of the denominator of
$C$ in (17) vanishes, that is,

$\left(m_{1} \cos m_{1} h_{0}-i m_{2} \sin m_{1} h_{0}\right) \exp \left(i m_{2} h_{0}\right)$

$$
+\alpha\left(m_{1} \cos m_{1} h_{0}+i m_{2} \sin m_{1} h_{0}\right) \exp \left[i m_{2}\left(2 H-h_{0}\right)\right]=0
$$

Necessary conditions for amplification are then

$$
m_{1}=\frac{\pi(2 q+1)}{2 h_{0}} \quad m_{2}=\frac{\pi(p+1)}{H-h_{0}}
$$

or

$$
m_{1}=\frac{\pi(q+1)}{h_{0}} \quad m_{2}=\frac{\pi(2 p+1)}{2\left(H-h_{0}\right)}
$$


SHEARWATER FEB 22 1986, 13:27 GMT

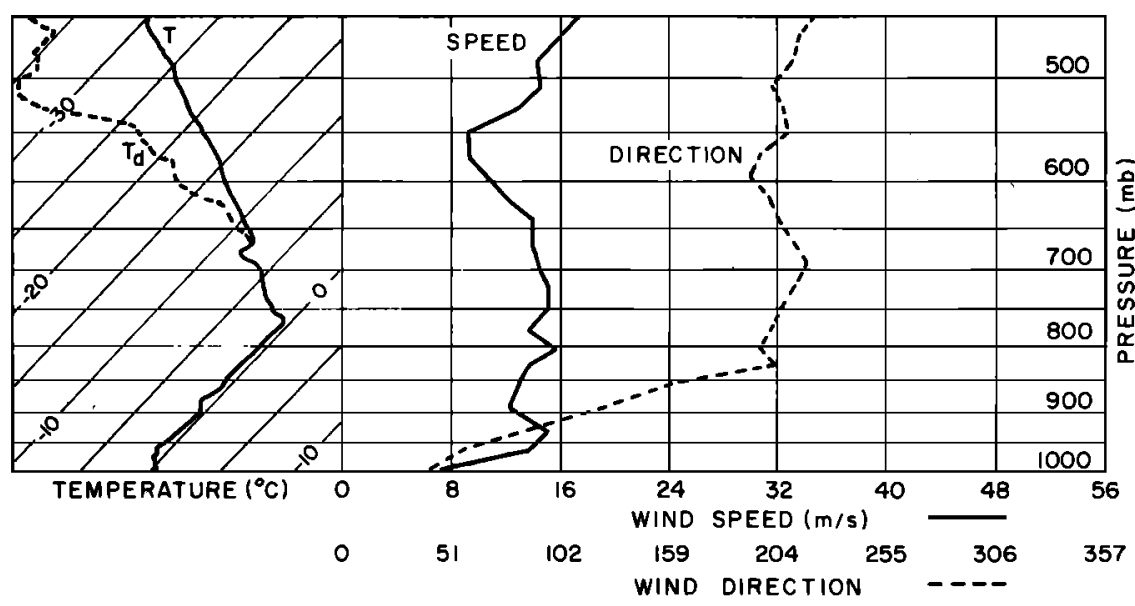

Fig. 13. Soundıngs for temperature $T$, dew-point temperature $T_{d}$, wind speed, and direction at Shearwater, Nova Scotia, February 22, 1986, at 1318 UT (GMT).

with $p, q=0,1,2,3, \cdots$. If any of these two sets of conditions (38) are satisfied, the atmosphere is then tuned for maximum response as $\alpha$ varies from zero to unity, with the largest amplitude occuring at $\alpha=1$. Recall that the parameter $\alpha=\left(m_{2}\right.$ $\left.-m_{3}\right) /\left(m_{2}+m_{3}\right)$ represents the coefficient of reflection of vertically propagating gravity waves at the interface between two layers with vertical wave numbers $m_{2}$ and $m_{3}$, respectively. These amplification conditions, derived above for a three-layer atmosphere, -reduce to the following for a two-layer atmosphere:

$$
m_{1}=\frac{\pi(2 n+1)}{2 h_{0}}
$$

with $n=0,1,2, \cdots$. This is identical to the condition for resonance and ducting of hydrostatic waves as found by Lindzen and Tung [1976]. This resonant condition can be easily understood in terms of the two-layer model: when the vertical wave number in the bottom layer is larger than that in the top layer, the coefficient of reflection is nonzero and vertically propagating gravity waves would be partially reflected downward at the interface $\boldsymbol{H}$. Depending on the number of quarter wavelengths between the ground and the discontinuity, there is interference with the reflected waves, and the wave amplitude may be enhanced in the steady solution. For the threelayer model, two different sets of "resonance" conditions are possible, as shown in (38). For the zero mode $(p=q=0)$ resonance to occur, we need the bottom layer thickness to coincide with a quarter wavelength of the perturbation and a half wavelength in the middle layer. The other possibility is to have a half wavelength in the bottom layer and a quarter wavelength in the middle layer.

In Table 1 we show the amplification of the maximum vertical velocity $\left(w_{\max }\right)$ below the interface $z=H$ for a three-layer model, as the coefficient of reflection $\alpha$ varies from zero to unity. The amplification factor, $A$, is defined as the amplitude of maximum response normalized by the amplitude obtained with $\alpha=0$ (i.e., when $m_{1}=m_{2}=m_{3}$ ). Figure 11 shows the "resonant" amplification of the vertical velocity as a function of the nondimensional vertical wave number of the lowest layer $\left(m_{1} h_{0}\right)$. For an atmosphere with no wind shear, the latter quantity is also the inverse thermally induced Froude number of the bottom layer. The peak is associated with the zeromode $(p=q=0)$ "resonance" in $(38 b)$; the value of the reflection coefficient is $\alpha=0.75$. The amplitude would be reduced in the atmosphere due to the presence of viscosity.

Figure 12 shows the change in the vertical motion field $w(x$, $z$ ), as the reflection coefficient is varied from zero to unity. For $\alpha=0,0.4,0.6$ (Figures $12 a, 12 b$, and $12 c$ ), the amplitude of the circulation is weak. The amplitude for $\alpha=0.8$ is significantly enhanced. However, the limit $\alpha=1$ is not considered here, as nonhydrostatic effects become important and a new discrete spectrum of horizontal wave numbers becomes resonant; for example, lee waves appear. Scorer [1949] first treated this problem for a vertical wave number which varies strongly with altitude and showed that a rapid decrease of the vertical wave number with height produces trapped lee waves. Al-

VERTICAL WAVE NUMBER VS PRESSURE SHEARWATER FEB 22,1318 GMT 1986

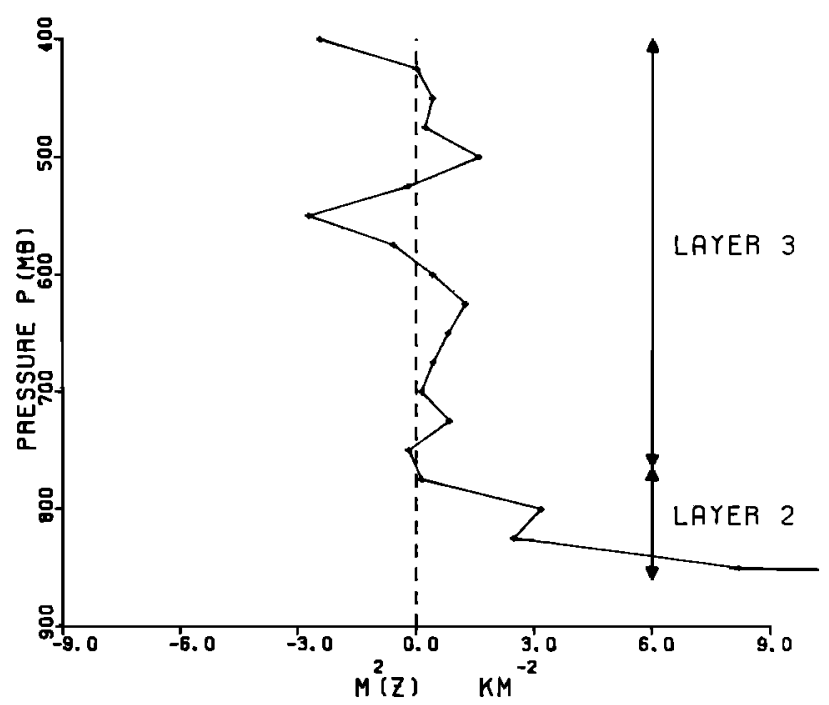

Fig. 14. Square of the vertical wave number $\left(\mathrm{m}^{2}\right)$ as a function of pressure, for the soundings of Figure 13. 
SHEARWATER FEB 5 1986, 17:18 GMT

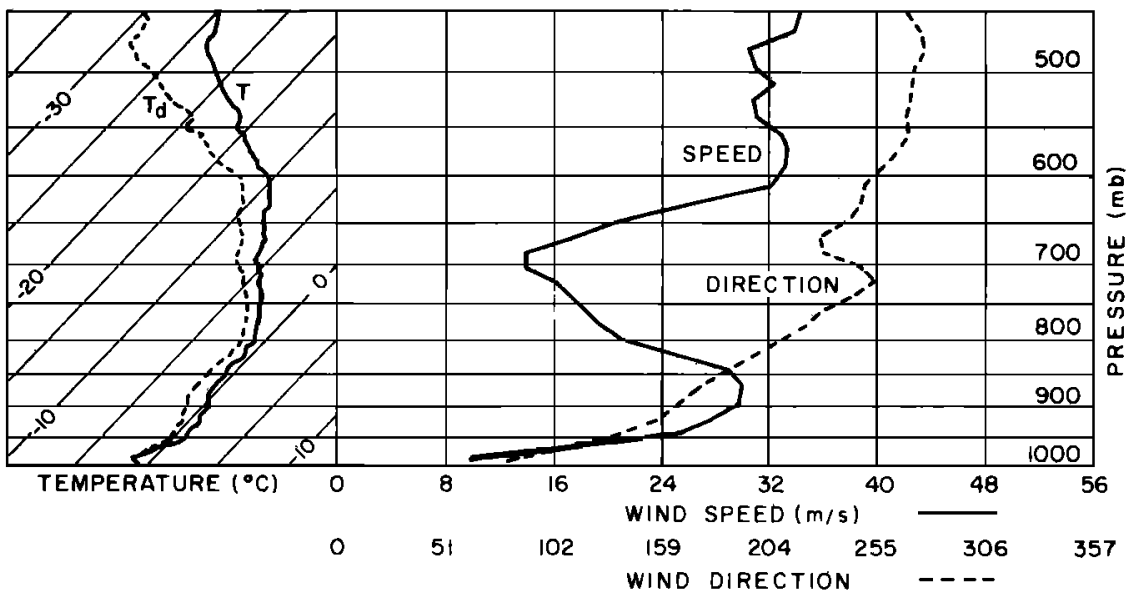

Fig. 15. As in Figure 13, but for February 5, 1986, at 1718 UT (GMT).

though these results were originally derived for flow over topography, they also apply to flow over a heat source, as the governing equations are similar in both cases. We note that in terms of our model formulation, the hydrostatic approximation can be expressed as [Smith, 1980]

$$
m_{i} a \gg 1
$$

where $i=1,2,3$ is the layer index and $a$ is a horizontal scale characterizing the heat source dimension. The trapped resonant horizontal waves which appear in the nonhydrostatic regime, as described by Scorer [1949], probably do not play a major role in the amplification mechanism examined in this study. This is supported by numerical simulations of topographically forced flows by Durran [1986], which suggest that lee waves are small-scale features superposed on the forcing hydrostatic flow. The latter is dominant as long as the hydrostatic condition (40) holds. When $m_{i} a$ approaches unity, superposed lee waves would appear, but they are unlikely to qualitatively change the nature of the amplification mechanism.

We also see from Figure 12 that the isopleths in the top layer $(z>2.57 \mathrm{~km})$ display an upstream tilt for low values of $\alpha$ (Figures $12 a$ and $12 b$ ), which becomes progressively vertical as $\alpha$ tends to unity. This in turn means that the downward momentum flux $\left(u^{\prime} w^{\prime}\right)$, which depends on the vertical tilt, decreases as $\alpha$ approaches unity. We note that in Figure $12 d$ the depth of the lower layer $\left(h_{0}=1.57 \mathrm{~km}\right)$ is such that it contains a half wavelength of the perturbation, whereas the middle layer between $z=1.57 \mathrm{~km}$ and $z=2.57 \mathrm{~km}$ contains a quarter wavelength. This is consistent with the "resonance" conditions (38) discussed earlier. These conditions, derived for an elevated diabatic source, remain unchanged for a surface diabatic forcing. There are other physical analogues of these resonant modes. For example, the normal modes of a rope fixed at one end $(z=0)$ and free to move at the other end $\left(z=h_{0}\right)$, are identical to those for vertical standing gravity waves in our two-layer model atmosphere (i.e., equation (39)).

Our analysis of the amplification mechanism due to partial reflection from the layer interface $z=H$ is similar to the analysis of Klemp and Lilly [1975] of wave-induced downslope winds for flow over topography. One difference is that the effect of the stratosphere is included in their analysis. However, removing the stratosphere, which is the top layer in their three-layer atmosphere, would still produce an amplification of standing vertical buoyancy waves. In this case, amplification in the troposphere still results when the static stability of the second layer is much smaller than that of the bottom layer. Durran [1986], in his analysis of downslope windstorms, also showed that a significant response in the troposphere is still produced without the change in static stability at the tropopause. In our analysis, amplification is produced by a change in static stability due to the presence of a near- $0^{\circ} \mathrm{C}$ melting layer. Both the melting layer and the reflecting interface are typically located in the lower troposphere. The presence of a stratosphere is thus not crucial in the amplification mechanism discussed in this study. In section 7, we use data obtained from Canadian Atlantic Storms Program (CASP) to examine the potential importance of this mechanism for precipitation enhancement.

\section{VERTICAL WAVE NUMBER VS PRESSURE
SHEARWATER 1718 GMT FEB 51986}

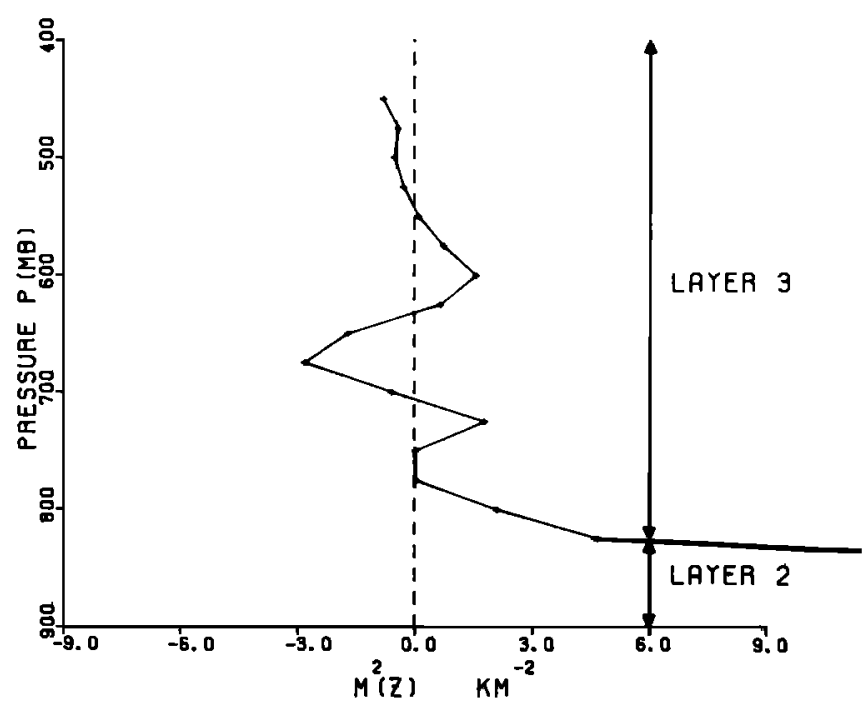

Fig. 16. As in Figure 14, but for the soundings of Figure 15. 
BUFFALO DEC $61983,1200 \mathrm{GMT}$

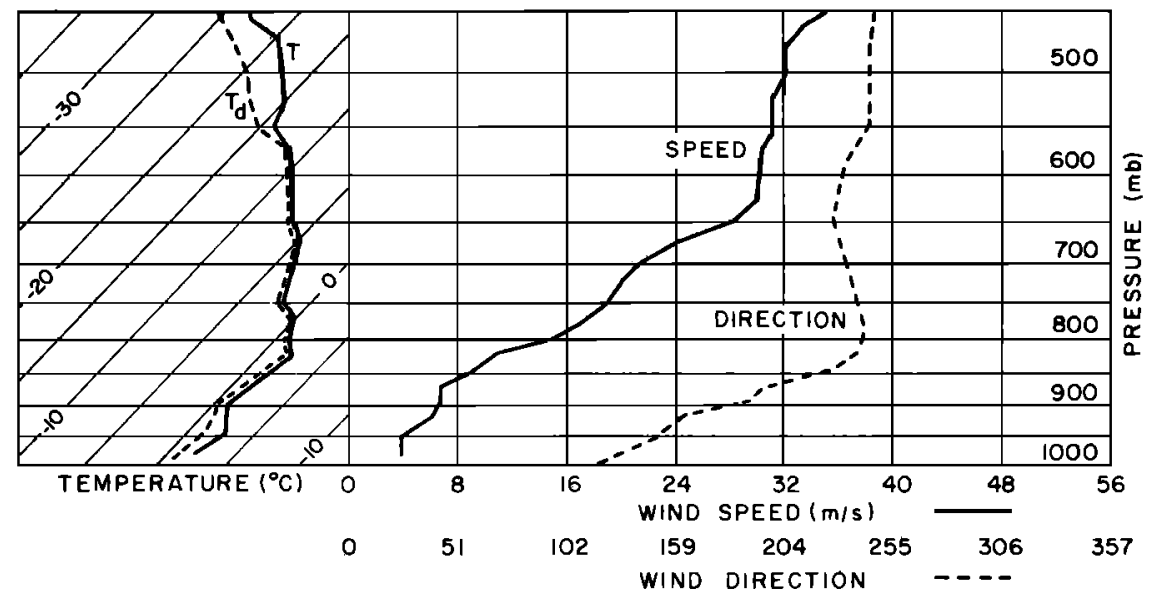

Fig. 17. As in Figure 13, but for Buffalo, New York, May 13, 1984, at 1200 UT (GMT) [from Stewart and King, 1986].

\section{Data From Canadian Atlantic Storms Program}

We now examine the possible application of the theory to the atmosphere with a melting-induced elevated diabatic forcing. We investigate two cases during CASP [Stewart et al., 1987] and one case over southern Ontario and surrounding areas [Stewart and King, 1987]. Rain/snow boundaries and melting are present in all these cases. Two of the three cases are examples of deep near $-0^{\circ} \mathrm{C}$ isothermal layers. The first case period occured from 0600 UT on February 22, 1986, to 0600 UT on February 23, 1986 (CASP, IOP \#9). Dry bulb temperatures were above zero at the surface after 0900 UT. Shortly after that time, when melting started, precipitation was enhanced and heavy rain was recorded for nearly 3 consecutive hours (1000-1300 UT). The sounding at Shearwater, Nova Scotia (Figure 13) shows a deep near- $0^{\circ} \mathrm{C}$ isothermal layer below 760 mbar. A nearly conditionally unstable layer caps the melting layer.

To calculate the structure of the vertical wave number the sounding can be divided into three layers. The top layer has a temperature profile, which is close to the moist adiabatic lapse rate, and a wind profile, which has a positive curvature $\left(\partial^{2} \bar{U} / \partial z^{2}>0\right)$. This yields a small value for $m_{3}$, according to (6). The middle layer is the melting layer located between about 760 and $850 \mathrm{mbar}$ and shows little curvature of the wind profile $\left(\partial^{2} \bar{U} / \partial z^{2} \approx 0\right)$. The resulting $m_{2}$ value is large because of relative stronger static stability. Finally, the third layer lies below 850 mbar, but no attempt was made to compute the vertical wave number because of the strong directional change of the wind speed. Both $m_{2}$ and $m_{3}$ are calculated using (6). Compressibility and moisture effects have been included in the calculation of the static stability for a moist atmosphere $\left(N_{w}\right)$, using the following expression from Lalas and Einaudi [1974]:

$$
N_{w}{ }^{2}=-g\left(\frac{1}{\rho_{m 0}} \frac{d \rho_{m 0}}{d z}+\frac{g}{c_{w}^{2}}\right)
$$

where $\rho_{m 0}=\rho_{0}+\rho_{w v}$ is the total air density including both dry air $\left(\rho_{0}\right)$ and water vapor $\left(\rho_{w v}\right) ; c_{w}$ is the speed of sound in a moist atmosphere. Equation (41) gives a more accurate representation of the static stability when the latter is close to moist adiabatic. The value of $m$ was calculated at every 25- mbar interval, using (6) and (41); a smoothing 1-2-1 operator was also applied. The sounding for the first case period (Shearwater, February 22, 1986) and the resulting structure of the computed vertical wave number are shown in Figures 13 and 14 , respectively. The corresponding quantities for the second case period during CASP (Shearwater, February 5, 1986, 1718 UT, CASP IOP \#6) are shown in Figures 15 and 16. There is again a near $-0^{\circ} \mathrm{C}$ isothermal layer, occuring between 800 and 975 mbar. The results for the final case are shown in Figures 17 and 18. This case (Buffalo, December 6, 1983,1200 UT) is taken from Stewart and King [1986], and is again characterized by melting. A summary of the results for the three cases, showing the average values of $m_{2}$ and $m_{3}$ computed from the soundings, as well as the corresponding values required for resonant amplification, is contained in Table 2. The comparison shows that an enhanced response due to a discontinuity in the vertical wave number is quite

VERTICAL WAVE NUMBER VS PRESSURE

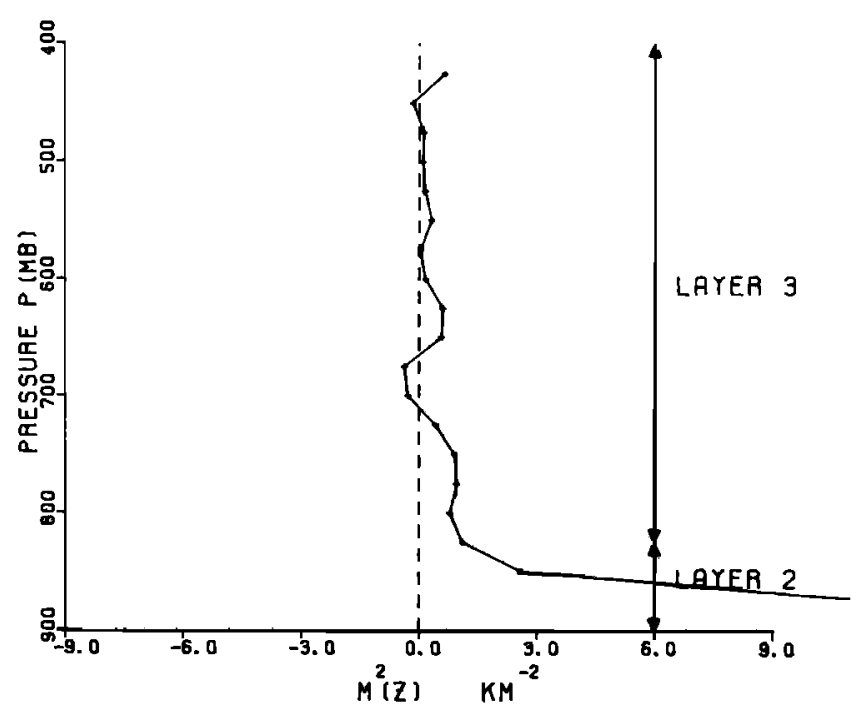

Fig. 18. As in Figure 14, but for the soundings of Figure 17. 
TABLE 2. Results of Case Studies Compared to Theory

\begin{tabular}{|c|c|c|c|c|c|c|c|}
\hline \multirow[b]{2}{*}{ Case } & \multirow[b]{2}{*}{ Date } & \multirow[b]{2}{*}{ Location } & \multicolumn{4}{|c|}{ Computed From Sounding } & \multirow{2}{*}{$\begin{array}{c}\begin{array}{c}\text { Obtained } \\
\text { From Theory }\end{array} \\
m_{2}\left(H-h_{o}\right)\end{array}$} \\
\hline & & & $m_{2}\left(H-h_{\mathrm{o}}\right)$ & $m_{2}$ & $m_{3}$ & $\alpha$ & \\
\hline $\begin{array}{l}\text { CASP IOP } \\
\# 9\end{array}$ & Feb. 22, 1986 & $\begin{array}{l}\text { Shearwater, Nova } \\
\text { Scotia }\end{array}$ & 1.72 & 1.87 & 0.02 & 0.98 & $\begin{array}{c}\pi / 2 \\
(p=0)\end{array}$ \\
\hline $\begin{array}{l}\text { CASP IOP } \\
\# 6\end{array}$ & Feb. 5, 1986 & $\begin{array}{l}\text { Shearwater, Nova } \\
\text { Scotia }\end{array}$ & $3.38^{*}$ & $4.93 *$ & 0.54 & 0.80 & $\begin{array}{c}\pi \\
(p=0)\end{array}$ \\
\hline SRN Ontario & $\begin{array}{r}\text { Dec. 6, } 1983 \\
(1200 \text { UT })\end{array}$ & southern Ontario & $2.6^{*}$ & $3.67^{*}$ & 0.55 & 0.74 & $\begin{array}{c}\pi \\
(p=0)\end{array}$ \\
\hline
\end{tabular}

*Values calculated using winds with rapidly changing direction in the vertical in layer 2 ; these results may thus be only qualitative.

possible. This discontinuity results from the large change in static stability due to the presence of a near $-0^{\circ} \mathrm{C}$ isothermal layer. This is an important result, because melting plays an important role in the formation of deep $0^{\circ} \mathrm{C}$ isothermal layers and other mesoscale circulations [Stewart, 1984; Szeto et al., $1988 \mathrm{~b}]$. Thus the amplification mechanism identified in this study is potentially an important feedback mechanism in precipitation enhancement associated with melting.

\section{CONClusions}

In this paper we have derived analytic solutions describing the nature of two-dimensional airflow over diabatic sources. In particular, we examine the response to elevated cooling sources due to melting. The latter frequently produces an isothermal layer capped by another layer of significantly weaker static stability, resulting in a strong variation in the vertical of the vertical wave number $m(z)$. This variation is known to be important for airflow over topography.

A piecewise uniform model for $m(z)$ is used here to examine the effects of partial reflections from a layer interface. The results show that an amplification of the response due to diabatic sources may be obtained. An analysis of CASP sounding data associated with melting shows that a two- or three-layer model is useful in investigating this amplification mechanism. This resonance mechanism is potentially important for precipitation enhancement in melting-induced mesoscale circulations.

We also show that the resonance conditions for a surface diabatic source are similar to those for an elevated source; this means simultaneous resonance is possible. This would be important when the combined effects of surface and elevated diabatic forcings are considered; the former might be associated with heating over the Gulf Stream regions, and the latter due to melting. In linear theory the combined response is simply the sum of the responses due to the individual diabatic sources. A more sophisticated nonlinear model could significantly modify the linear response. Durran [1986] has shown that linear theory may not provide an accurate representation of the nonlinear response in the simulation of mountain waves in multilayer atmospheres. The prescribed diabatic forcings used in our study also do not allow feedbacks between the forced mesoscale circulations and the precipitating system. A coupled dynamical-microphysical numerical model is needed to further explore this important aspect.

The analytical solutions derived in our study are useful in identifying and clarifying dynamical mechanisms; they may also be used to verify the results from more sophisticated models. The results from our simple model demonstrate the effects of vertical variation of $m(z)$ associated with melting; a potentially important amplification mechanism is also identified. Our results thus provide strong motivation for further numerical experiments to study melting induced mesoscale circulation.

\section{APPENDIX A}

The pressure must be continuous across the interface between layers $i$ and $i+1$.

$$
p_{i}^{\prime}=p_{i+1}{ }^{\prime}
$$

Integrating (1) with respect to $x$ and using (3), gives

$$
p^{\prime}=\rho_{0} \bar{U} \int \frac{\partial w^{\prime}}{\partial z} d x-\rho_{0} \frac{d \bar{U}}{d z} \int w^{\prime} d x
$$

Using (A1) and (A2), we obtain

$\bar{U}_{i} \int \frac{\partial w_{i}^{\prime}}{\partial z} d x-\frac{d \bar{U}_{t}}{d z} \int w_{i}^{\prime} d x$

$$
=\bar{U}_{i+1} \int \frac{\partial w_{i+1}{ }^{\prime}}{\partial z} d x-\frac{d \bar{U}_{i+1}}{d z} \int w_{i+1}^{\prime} d x
$$

Differentiating with respect to $x$ and using the continuity conditions $\bar{U}_{i}=\bar{U}_{i+1}$ and $w_{z}=w_{i+1}$ at the interface $z=h_{0}$ or $z=H$ lead to the following interface condition:

$$
\frac{\partial w_{i}^{\prime}}{\partial z}=\frac{\partial w_{i+1}{ }^{\prime}}{\partial z}+\frac{1}{\bar{U}_{i}}\left[\frac{d \bar{U}_{i}}{d z}-\frac{d \bar{U}_{i+1}}{d z}\right] w_{i}^{\prime}
$$

If we neglect the discontinuity of the basic wind shear across the interface, we obtain the following condition at the interfaces $z=h_{0}, H$.

$$
\frac{\partial w_{\imath}^{\prime}}{\partial z}=\frac{\partial w_{i+1}^{\prime}}{\partial z}
$$

which equals conditions 5 and 6 (equation (15)) in the text. Note that (A5) represents the continuity of pressure when there is no discontinuity in wind shear across the interface.

\section{ÁPPENDIX B}

The scaling of the first term on the left-hand side of (8) is $W / D^{2}$, where $D$ is the vertical scale of the heating. The first term can thus be neglected compared to the second, if

$$
\frac{1}{D^{2}} \ll m^{2}
$$

that is, when the thermally induced Froude number $(F r)$ is 
much smaller than unity.

$$
F r=\frac{1}{m D}=\frac{U}{N D} \ll 1
$$

When this condition holds, we have a positive phase relationship between the vertical velocity $(w)$ and diabatic forcing $(J)$. This result can also be obtained by setting $U=0$ in (4). Conversely, when $F r \gg 1,(8)$ reduces to

$$
\frac{\partial^{2} w}{\partial z^{2}}=\frac{g J_{0} G(x) F(z)}{C_{p} \bar{T} \bar{U}^{2}}
$$

Assuming a sinusoidal structure for $F(z)$, we obtain the result that the vertical velocity is exactly out of phase with the heating.

\section{APPENDIX C}

We derive here the solution for flow over an elevated heat source having the following vertical profile

$$
\begin{array}{ll}
F(z)=1 & z_{H}-d / 2<z<z_{H}+d / 2 \\
F(z)=0 & \text { otherwise }
\end{array}
$$

The elevated source is centered at $z=z_{H}$ and has a depth $d$. The Green's function method is more appropriate here for the given function $F(z)$. We seek the solution for a two-layer model for $m$. When $z_{H}+d / 2<h_{0}$, with $h_{0}$ being the height of the discontinuity of $m$, the solution for the vertical velocity is

$$
\begin{aligned}
& w(x, z)=\frac{2 A A}{m_{1}}\left\{a\left[\left(1+\alpha^{2}\right) \cos m_{1} z_{H}+2 \alpha \cos m_{1}\left(z_{H}-2 h_{0}\right)\right]\right. \\
& \left.+x\left(x^{2}-1\right) \sin m_{1} z_{H}\right\} \sin m_{1} z \sin m_{1} d / 2 \quad z<z_{H}-d / 2 \\
& w(x, z)=\frac{A A}{m_{1}}\left\{a\left[\left(1+\alpha^{2}\right) \cos m_{1} z+2 \alpha \cos m_{1}\left(z-2 h_{0}\right)\right]\right. \\
& \left.-x\left(1-\alpha^{2}\right) \sin m_{1} z\right\}\left\{\cos m_{1}\left(z_{H}-d / 2\right)-\cos m_{1} z\right\} \\
& +\frac{A A \sin m_{1} z}{m_{1}}\left\{a \left[\left(1+\alpha^{2}\right)\left(\sin m_{1}\left(z_{H}+d / 2\right)-\sin m_{1} z\right)\right.\right. \\
& \left.+2 \alpha\left(\sin m_{1}\left(z_{H}+d / 2-2 h_{0}\right)-\sin m_{1}\left(z-2 h_{0}\right)\right)\right] \\
& \left.+x\left[\left(\alpha^{2}-1\right)\left(\cos m_{1} z-\cos m_{1}\left(z_{H}+d / 2\right)\right)\right]\right\} \quad z_{H}-\frac{d}{2} \leq z \leq z_{H}+\frac{d}{2} \\
& w(x, z)=\frac{2 A A}{m_{1}}\left\{a\left[\left(1+\alpha^{2}\right) \cos m_{1} z+2 \alpha \cos m_{1}\left(z-2 h_{0}\right)\right]\right. \\
& \left.-x\left(1-\alpha^{2}\right) \sin m_{1} z\right\} \sin m_{1} z_{H} \sin m_{1} d / 2 \quad z_{H}+d / 2<z \leq h_{0} \\
& w(x, z)=\frac{2 A A}{m_{1}}\left\{a \left[(\alpha+1) \cos \left(m_{1} h_{0}-m_{2} h_{0}+m_{2} z\right)\right.\right. \\
& \left.+\alpha(\alpha+1) \cos \left(m_{1} h_{0}+m_{2} h_{0}-m_{2} z\right)\right] \\
& -x\left[(\alpha+1) \sin \left(m_{1} h_{0}-m_{2} h_{0}+m_{2} z\right)\right. \\
& \left.\left.+\alpha(\alpha+1) \sin \left(m_{2} z-m_{1} h_{0}-m_{2} h_{0}\right)\right]\right\} \\
& \cdot \sin m_{1} z_{H} \sin m_{1} d / 2 \quad z>h_{0}
\end{aligned}
$$

with

$$
A A=\frac{Q_{0} a g}{C_{p} \bar{T} \bar{U}^{2} m_{1}\left(1+\alpha^{2}+2 \alpha \cos 2 m_{1} h_{0}\right)\left(a^{2}+x^{2}\right)} \quad \alpha=\frac{m_{1}-m_{2}}{m_{1}+m_{2}}
$$

It is easy to show that the denominator of $A A$ goes to zero when $\alpha=1$ and when $2 m_{1} h_{0}=\pi(2 n+1)$. This is the condition already found in section 6 for a two-layer atmosphere. Notice, however, that "non-singular resonance" is not present here, because the function $F(z)$ used earlier is nonsinusoidal. If the diabatic source is located in the top layer $\left(z_{H}-d / 2>h_{0}\right)$, the resonance condition remains unchanged.

\section{NOTATION}

$a$ half width of heating source profile.

$a_{0}$ scale of compensative diabatic source.

$A$ amplification factor.

$c$ horizontal scale of surface diabatic forcing.

$C_{p}$ specific heat capacity of air.

$c_{0}$ horizontal scale of compensative diabatic forcing.

$c_{w}$ speed of sound in moist atmosphere.

$d_{e}$ distance over which evaporation occurs.

$d_{m}$ distance over which melting occurs.

$F(z)$ vertical structure function for heating.

$G(x)$ horizontal structure function for elevated heat source.

$G_{s}(x)$ horizontal structure function for surface heat source.

$g$ gravitational acceleration.

$H$ height at the top of middle layer.

$h_{0}$ height at the top of first layer.

$H_{0}$ heat flux.

$J$ heating rate.

$k$ horizontal wave number.

$L_{f}$ latent heat of fusion.

$m$ vertical wave number.

$M$ vertical scale of elevated heat source.

$N_{w}$ Brunt-Väisälä frequency in moist atmosphere.

$P_{0}$ precipitation rate.

$p^{\prime}, p$ pressure perturbation.

$J_{0}$ amplitude of heating rate.

$\bar{T}$ temperature of basic state.

$u^{\prime}, u$ wind perturbation.

$\bar{U}$ mean basic wind.

$w^{\prime}, w$ perturbation vertical velocity.

$x, y, z$ spatial coordinates.

$\alpha$ coefficient of reflection of internal gravity waves.

$\rho^{\prime}, \rho$ perturbation density.

$\rho_{0}$ air density of basic state.

$\rho_{i}$ density of ice.

$\rho_{m o}$ air density in moist atmosphere.

$\rho_{w v}$ density of water vapor.

$\Psi$ stream function.

$\zeta$ streamline displacement.

Acknowledgments. Comments and suggestions from two anonymous reviewers which led to an improvement of the manuscript are gratefully acknowledged. A. Cossette provided expert typing assistance. The CASP data were kindly made available by $\mathbf{R}$. Stewart. This work is supported by the Natural Sciences and Engineering Research Council (NSERC), and the Atmospheric Environment Service (AES).

\section{REFERENCES}

Atlas, D., R. Tatehira, R. C. Srivastava, W. Marker, and R. E. Carbone, Precipitation-induced mesoscale wind perturbations in the melting layer, Q. J. R. Meteorol. Soc., 95, 544-560, 1969.

Bretherton, C., Group velocity and the linear response of stratified fluids to internal heat or mass sources, J. Atmos. Sci., 45, 81-93, 1988.

Carbone, R. E., A severe frontal rainband, 1, Stormwide hydrodynamic structure, J. Atmos. Sci., 39, 258-279, 1982. 
Durran, D. R., Another look at downslope windstorms, I, J. Atmos. Sci., 43, 2527-2543, 1986.

Fernandez, W., A review of downdrafts at the rear of tropical squall lines, Bull. Am. Meteorol. Soc., 63, 1285-1293, 1982.

Fujita, T., Study of mesosystems associated with stationary radar echoes, J. Meteorol., 16, 38-52, 1959.

Gıll, A. E., Atmosphere-Ocean Dynamics, 662 pp., Academic, San Diego, Calif., 1982.

Hayashi, Y., Non-singular resonance of equatorial waves under radiation condition, J. Atmos. Scl., 33, 183-201, 1976.

Hsu, H. M., Study of linear steady atmospheric flow above a finite surface heatıng, J. Atmos. Sci., 44, 186-199, 1987.

Klemp, J. B., and D. K. Lilly, The dynamics of wave-induced downslope winds, J. Atmos. Sct., 32, 320-339, 1975.

Lalas, D., and F. Einaudi, On the correct use of the wet adiabatic lapse rate in stability of a saturated atmosphere, J. Appl. Meteorol., $11,318-324,1974$.

Lin, C. A., and R. E. Stewart, Mesoscale circulations initiated by melting snow, J. Geophys. Res., 91, 13,299-13,302, 1986.

Lin, Y. L., and R. B. Smith, Transient dynamics of airflow near a local heat source, J. Atmos. Sci., 43, 40-49, 1986.

Lindzen, R. S., and K. K. Tung, Banded convective activity and ducted gravity waves, Mon. Weather Rev., 104, 1602-1617, 1976.

Queney, P., The problem of airflow over mountains: A summary of theoretical studies, Bull. Am. Meteorol. Soc., 29, 16-26, 1948.

Raymond, D. J., Calculation of airflow over an arbitrary ridge including diabatic heating and cooling, J. Atmos. Sci., 29, 837-843, 1972.

Raymond, D. J., Wave-CISK, in Dynamics of Mesoscale Weather Systems, NCAR Summer Colloq. Lect. Notes, edited by J. B. Klemp, National Center for Atmospheric Research, Boulder, Colo., 1984.

Rotunno, R., On the linear theory of the land and sea breeze, $J$. Atmos. Sci., 40, 1999-2009, 1983.
Scorer, R. S., Theory of waves in the lee of mountains, Q. J. R. Meteorol. Soc., 75, 41-56, 1949.

Smith, R. B., Linear theory of stratified hydrostatic flow past an isolated mountain, Tellus, 32, 348-364, 1980.

Smith, R. B., and Y. L. Lin, The addition of heat to a stratified airstream with application to the dynamics of orographic rain, $Q . J$. R. Meteorol. Soc., 108, 353-378, 1982.

Stewart, R. E., Deep $0^{\circ} \mathrm{C}$ isothermal layers within precipitation bands over southern Ontario, J. Geophys. Res., 89, 2567-2572, 1984.

Stewart, R. E., and P. King, Rain-snow boundaries over southern Ontario, Mon. Weather Rev., 115, 1894-1907, 1987.

Stewart, R. E., R. W. Shaw, and G. A. Issac, Canadian Atlantic Storms Program: The meteorological field project, Bull. Am. Meteorol. Soc., 68, 338-345, 1987.

Szeto, K. K., C. A. Lin, and R. E. Stewart, Mesoscale circulations forced by melting snow, I, Basic simulations and dynamics, $J$. Atmos. Sci., 45, 1629-1641, 1988.

Szeto, K. K., R. E. Stewart, and C. A. Lin, Mesoscale circulations forced by melting snow, II, Application to meteorological features, J. Atmos. Sci., 45, 1642-1650, 1988.

C. A. Lin and A. Robichaud, Climate Research Group, Department of Meteorology, McGill University, 805 Sherbrooke Street West, Montreal, Quebec, Canada H3A 2K6.

(Received October 1, 1987;

revised October 28, 1988;

accepted November 3,1988 .) 